\title{
An urological cause of hypoglycaemia: A case report of the Doege-Potter syndrome
}

\author{
Nuno Ramos ${ }^{1}$, Rodrigo Ramos ${ }^{2}$, Celso Marialva ${ }^{2}$, Eduardo Silva ${ }^{2}$ \\ ${ }^{1}$ Urology Department, Garcia de Orta Hospital, Almada, Portugal; \\ ${ }^{2}$ Urology Department, Portuguese Institute of Oncology, Lisbon, Portugal.
}

\begin{abstract}
Summary The Doege-Potter syndrome is a rare paraneoplastic syndrome presenting with hypoglycaemia due to ectopic secretion of insulin-like growth factor II (IGF-II) from a solitary fibrous tumor. The underlying tumor can be benign or malignant and rarely present in extrapleural sites. We describe the case of a 83-year-old male diagnosed with a Doege-Potter syndrome due to a kidney tumor.
\end{abstract}

KEY WORDS: Hypoglycaemia; Insulin-like growth factor; Solitary fibrous tumor.

Submitted 19 June 2020; Accepted 24 July

\section{INTRODUCTION}

Solitary fibrous tumor (SFT) was first reported by Klemperer and Rabin in 1931 as a lesion that originated in the pleura $(1,2)$. Nevertheless, SFTs are rare mesenchymal tumors, found in various anatomic locations, with approximately $20 \%$ in extrapleural locations like liver, orbit, lung and gastrointestinal tract. These tumors occur mainly in adults, with a peak age of $60-80$ years and are equally distributed between genders (3). SFTs are rarely symptomatic and the vast majority are incidentally found. However, these tumors may also potentially cause symptoms, such as refractory hypoglycaemia, due to the production of insulin-like growth factor (IGF-II). When hypoglycaemia is associated with an SFT, it is referred as the Doege-Potter syndrome, a potentially life-threatening condition $(2,4)$. Majority of the tumors are benign, but 10\%-20\% can be malignant. Malignancy is determined by invasive growth with an unclear boundary, high cell density, mitoses, pleomorphism and the presence of bleeding or necrosis (5). Benign SFTs can be cured by complete surgical resection. The role of chemotherapy and radiotherapy is still unclear. We describe the case of an 83-year-old man, who underwent a surgical resection for a SFT, associated with refractory hypoglycaemia.

\section{Case Report}

A male 83 years old male patient was referred to our hospital due to a progressive increase of abdominal volume, in association with frequent episodes of syncope, as a result of hypoglycaemia. The patient had a history of arterial hypertension and was medicated with lisinopril. Physical examination revealed the presence of a palpable mass in the left flank. On admission, his blood glucose level was 33 mg/dl (normal range 70-109 mg/dl), being administrated continuous glucose infusion and glucocorticoid. The hypothesis of insulinoma was ruled out by serum insulin level of $12.3 \mu \mathrm{UI} / \mathrm{mL}$ (normal $<28.0$ $\mu \mathrm{UI} / \mathrm{mL}$ ) and serum C-peptide level of $3.6 \mathrm{ng} / \mathrm{ml}$ (normal range 0.9-7.1 ng/ml). Plasma IGF-I and IGF-II levels were measured being respectively $29.00 \mathrm{ng} / \mathrm{mL}$ (normal: 55 $166 \mathrm{ng} / \mathrm{mL}$ ) and $458 \mathrm{ng} / \mathrm{mL}$ (normal: 288-736 ng/mL). The ratio IGF-II/IGF-I was higher than 10, suggestive of hypoglycaemia caused by non-islet cell tumor, the DoegePotter syndrome. Computed tomography (CT) demonstrated a large renal exophytic mass in the left kidney of 19 x $17 \times 18.7 \mathrm{~cm}$, heterogeneous, with little contrastenhancement, without signs of renal vein invasion neither areas of necrosis (Figure 1). Open left radical nephrectomy was performed. The postoperative course was uneventful, and hypoglycemic symptoms disappeared immediately after surgery.

Histopathological analysis revealed a tumor composed of spindle cells without atypia neither mitoses or areas of necroses. Immunohistochemical staining revealed a tumor positive for activators of transcription 6 (STAT6), CD34 and IGF-II, and negative for MDM2, AE1/AE3, EMA, S-100, SMA, and desmin (Figure 1). Based on the histological and immunohistochemical findings, a diagnosis of SFT of the kidney was made. The tumor secreted IGF II, which caused hypoglycaemia, but did not present malignant potential. On the first year of followup, the patient was observed in consultation every 3 months, remaining tumor-free based on CT scan at 6 and 12 months. Patient reported no episodes of hypoglycaemia during this period. Due to the rarity of this tumor, there are no specific guidelines available to monitor these patients. Therefore, we have used the European Association of Urology (EAU) guidelines for Renal Cell Carcinoma for subsequent follow-up.

\section{Conclusions}

SFTs can arise from a wide range of anatomic sites, both intrapleural or extrapleural, as the retroperitoneum. Refractory hypoglycaemia may occur in $4 \%$ of SFT and is reported as Doege-Potter syndrome (6). It is caused by the inappropriate secretion of a high molecular-weight form of IGF-II (HMW IGF-II). This molecule is an incompletely processed molecule of IGF-II, derived from the tumor, which fails to form a complex with IGF-binding protein- 

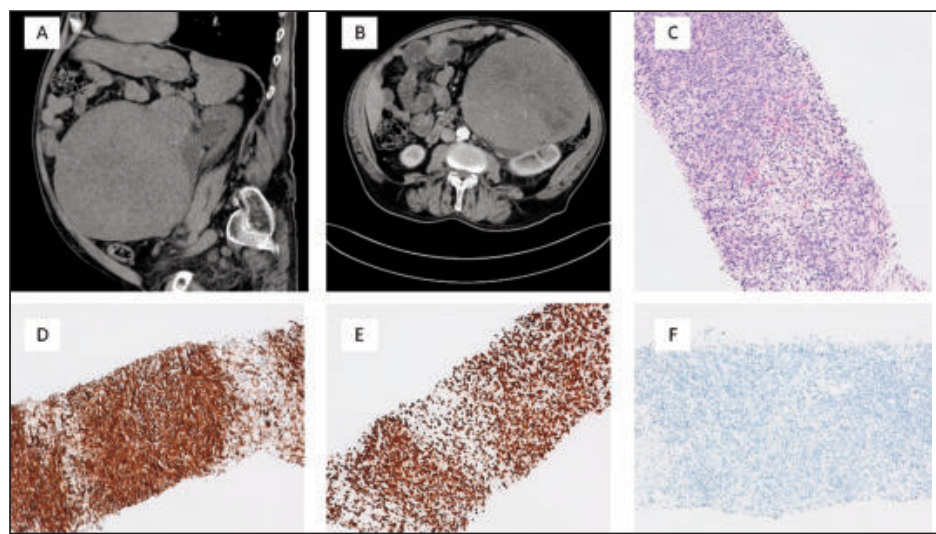

Figure 1.

Figure 1 Imaging, histopathological, and immunohistochemical findings.

(A, B) Computed tomography demonstrated a large tumor in the left kidney with little contrastenhancement, $(C)$ histopathological findings: the mass is a spindle cell tumor $(100 \times)(D, E, F)$ immunohistochemical findings:

(D) immunohistochemistry shows positive staining for CD34 (100x), (E) positive staining for STAT6 (100x), $(F)$ negative staining for $M D M 2, A E 1 / A E 3, E M A, S-$ 100, SMA, and desmin (100x).
3. This leads to higher bioavailability compared to mature IGF-II, with activation of insulin receptors, inhibiting hepatic gluconeogenesis and increasing peripheral glucose uptake which results in hypoglycaemia (7). The detection of HMW IGF-II requires immunoblot analysis, not available at our institute. The patients with Doege-Potter syndrome, usually presents suppressed serum insulin, $C$ peptide and low IGF-I against normal or elevated levels of IGF-II (8). CT scan and magnetic resonance imaging (MRI) are effective tools for identifying SFT, determining the invasion of the surrounding structures, revealing information on distant metastasis and for follow-up. CT imaging typically shows a smooth, well circumscribed, homogeneous mass with or without necrosis or calcifications (2). MRI presents as a tumor with high signal intensity on T2weighted images and iso-intensity or low intensity on T1weighted images (6). Fluorodeoxyglucose positron emission tomography (FDG-PET) can also offer benefit in the diagnosis but is less often used. Definitive diagnosis of an SFT depend upon histological identification of a spindle cell tumor with alternating areas of cellularity and hypocellular collagen rich areas, as well as consistent CD34 expression and negative expression patterns for cytokeratin AEl/AE3, desmin and S-100. Although the majority of SFTs are benign, 20\% may present local recurrence and metastases $(9,10)$. Malignant criteria include: hypercellularity (> 4 mitotic figures/10 high power fields), atypia, infiltrative growth pattern and necrosis (11). Complete tumor resection is the only definitive treatment. Due to its rarity, there are no guidelines for the treatment of metastatic or nonresectable SFT. Chemotherapy and consecutive selective embolization of the feeding vessels of the tumor can be tried to alleviate symptomatic hypoglycaemia in irresectable tumors, but they are not effective treatments. Radiotherapy has limited value, although it has been used as an adjuvant treatment in case of incomplete surgical resection $(3,12)$. Symptomatic medical treatment of hypoglycaemia has been tried with some success for patients unfit for surgery (8). In our patient, hypoglycaemia disappeared after the complete radical resection. However, the return of this symptom can be a sign of tumor recurrence, which can happen in an estimated rate of 9.9\% (2).

In conclusion Doege-Potter syndrome should be considered as a differential diagnosis in a patient with a known malignancy if accompanied by hypoglycaemia and SFTs have a favourable clinical outcome if complete resection is performed.

\section{References}

1. Wada Y, Okano K, Ando Y, et al. A solitary fibrous tumor in the pelvic cavity of a patient with Doege-Potter syndrome: a case report. Surg Case Rep. 2019; 5:60.

2. Forster C, Roumy A, Gonzalez M. Solitary fibrous tumor of the pleura with Doege-Potter syndrome: second recurrence in a 93-year-old female. SAGE Open Med Case Rep. 2019; 7:2050313X18823468.

3. Qian X, Zhou D, Gao B, Wang W. Metastatic solitary fibrous tumor of the pancreas in a patient with Doege-Potter syndrome. Hepatobiliary Surgery and Nutrition. 2020; 9:112-5.

4. Moreira BL, Monarim MA, Romano RF, et al. Doege-Potter syndrome. Radiol Bras. 2015; 48:195-6.

)5) Kim DW, Na KJ, Yun JS, Song SY. Doege-potter syndrome: a report of a histologically benign but clinically malignant case. J Cardiothorac Surg. 2017; 12:64.

6. Han G, Zhang Z, Shen X, et al. Doege-Potter syndrome: A review of the literature including a new case report. Medicine (Baltimore). 2017; 96:e7417.

7. Pant V, Baral S, Sayami G, Sayami P. Doege-Potter Syndrome, cause of nonislet cell tumor hypoglycemia: the first case report from Nepal. Int Med Case Rep J. 2017; 10:275-8.

8. Kalebi AY, Hale MJ, Wong ML, et al. Surgically cured hypoglycemia secondary to pleural solitary fibrous tumour: case report and update review on the Doege-Potter syndrome. J Cardiothorac Surg. 2009; 4:45.

9. Antonella D, Loren D, Maria C, et al. Doege-Potter syndrome by malignant solitary fibrous tumor of the liver: A case report and review of literature. World J Gastrointest Surg. 2019; 11:348-57.

10. Chen S, Zheng Y, Chen L, Yi Q. A broad ligament solitary fibrous tumor with Doege-Potter syndrome. Medicine (Baltimore). 2018; 97:e12564.

11. Fung EC, Crook MA. Doege-Potter syndrome and 'big-IGF2': a rare cause of hypoglycaemia. Ann Clin Biochem. 2011; 48:95-6.

12. Urbina-Lima AD, Roman-Martin AA, Crespo-Santos A, et al. Solitary Fibrous Tumor of the Urinary Bladder Associated with Hypoglycemia: An unusual case of Doege-Potter syndrome. Urol Int. 2019; 103:120-4.

\section{Correspondence}

Nuno Ramos, MD (Corresponding Author)

nuno.ramos@hgo.min-saude.pt

Garcia de Orta Hospital, Av. Torrado da Silva, 2801-951, Almada (Portugal)

Rodrigo Ramos, MD

Celso Marialva, MD

Eduardo Silva, MD

Urology Department, Portuguese Institute of Oncology, Lisbon (Portugal) 